\title{
IgD Myeloma with Two Types of Paraproteinemic Kidney Damage: Case Report
}

\author{
Elena Zakharova ${ }^{1 *}$ and Olga Vorobjova ${ }^{2}$ \\ ${ }^{1}$ Department of Nephrology, Moscow City Hospital n.a. S.P. Botkin \\ ${ }^{2}$ Department of Pathology, St-Petersburg National Centre of Clinical Morphology
}

Received: August 10, 2015; Accepted: August 25, 2015; Published: September 20, 2015

*Corresponding author: Elena Zakharova, Department of Nephrology, Moscow City Hospital n.a. S.P. Botkin, Tel: +79671346936; Fax: +74999451756; E-mail: helena.zakharova@gmail.com

\begin{abstract}
Clinical presentation of multiple myeloma and other lymphoproliferative disorders is characterised, beyond specific haematological signs and symptoms, by a broad spectrum of kidney lesions. Differential diagnostics is the major challenge, demanding pathology evaluation of kidney tissue, as different types of lesions, mainly paraproteinemic, cannot be differentiated solely on the basis of clinical features. Importantly, symptoms of kidney damage may dominate over LPD symptoms, and even preclude overt LPD's.
\end{abstract}

IgD myeloma is rare, but aggressive tumour, diagnosed approximately in 2-2.5\% cases of MM. Diagnostic problems arise from the fact, that the routine test does not detect the M-spike in $60 \%$ of patients, and when it is detected, the concentration is usually smaller than $2 \mathrm{~g} / \mathrm{dL}$. An overproduction of Light Chains (LC), usually $\lambda$, is found in $90-96 \%$ of patients. The disease course is often accompanied by AL amyloidosis and light chain proteinuria, renal failure is observed in $33 \%$ of cases at the moment of diagnosis.

The pathology pattern of renal damage in patients with LPD, showing a combination of cast-nephropathy and light chain deposition disease is also rare and reported in literature like single cases or small series.

We present here a case of IgD myeloma, manifested with combined kidney injury - light chain deposition disease and cast nephropathy.

Keywords: Myeloma; Immunoglobulin D; Cast Nephropathy; Light Chain Deposition Disease

\section{IgD Myeloma with Two Types of Paraproteinemic Kidney Damage: Case Report}

Clinical presentation of Multiple Myeloma (MM) and other Lymphoproliferative Disorders (LPD) is characterized, beyond specific haematological signs and symptoms, by a broad spectrum of kidney lesions. Renal involvement is defined by numerous mechanisms, but mainly by the deposition of secreted paraproteins. Paraproteinemic lesions, in turn, may be represented by organized (crystals, fibrils, microtubules), and non-organized deposition of monoclonal immunoglobulin's or fragments thereof, mostly light chains (LC). They may involve the four components of the kidney parenchyma: glomeruli, tubules, interstitium and blood vessels. Organized depositions include Cast Nephropathy (CN), light-chain tubulopathy, AL/AH amyloidosis, glomerulonephritis with an organized microtubular monoclonal deposits, and cryoglobulinemic Glomerulonephritis (GN). Non-organized deposition group comprise Light Chain/ Heavy Chain Deposition Disease (LCDD/HCDD), proliferative GN with monoclonal deposits of IgG/IgA, and non-proliferative GN with monoclonal deposits of IgM. It appears that amino acid sequence of the monoclonal LC and other monoclonal proteins, defining inherent biochemical properties, is the primary determinant of the specific pattern of renal parenchymal deposition and clinical disease. Patients may present with Acute Kidney Injury (AKI), Nephrotic Syndrome (NS), proteinuria and/ or haematuria, arterial hypertension or chronic kidney disease. Differential diagnostics is the major challenge, demanding pathology evaluation of kidney tissue, as the above mentioned lesions cannot be differentiated solely on the basis of clinical features. Importantly, symptoms of kidney damage may dominate over LPD symptoms, and even preclude overt LPD's [1-13].

MM presents with end organ damage manifested as one or more of the following -bone pain, kidney damage, impaired haematopoiesis, hypercalcemia, and susceptibility to infections. In laboratory tests MM typically manifests itself by the presence of high paraprotein (mainly IgG, IgA and Bence-Jones) levels in serum and urine. Sometimes, however, the clinical picture of MM is quite different from the classic manifestation, which can cause diagnostic difficulties, thereby delaying the treatment. One of these rare and unusual forms of MM is IgD myeloma.

Plasma cell leukemia with IgD paraprotein was firstly described in 1968, and since that time IgD myeloma remains rare, but aggressive tumour, diagnosed approximately in $2-2.5 \%$ cases of $\mathrm{MM}$ and affecting young people. Diagnostic problems arise from the fact, that the routine test does not detect the M-spike in $60 \%$ of patients, and when it is detected, the concentration is usually smaller than $2 \mathrm{~g} / \mathrm{dl}$. An overproduction of LC (BenceJones paraprotein), usually $\lambda$, is found in $90-96 \%$ of patients. 
The disease course is often accompanied by AL amyloidosis and LC proteinuria, renal failure is observed in 33\% of cases at the moment of diagnosis. Lymphadenopathy is seen in $10 \%$ of patients [14-21].

The pathology pattern of renal damage in patients with LPD, showing a combination of cast nephropathy and LCDD is also rare - such coexistence is reported in literature like single cases or small series. At least two possible explanations for these findings suggested so far. It was observed, that both fibrillar and non-fibrillar monoclonal LC deposits may coexist in the same patient, and the identity of the amino acid sequence of the deposited protein has been reported. On the other hand, cases with more than one pattern of LC deposition may be explained by the biclonal proliferative process with more than one pathogenic LC, causing damage. Again, only rare MM (2\%) result in biclonal gammopathy with the production of two different heavy chains and/or light chains, with the combinations of IgG/IgM, IgA/ IgG, $\kappa / \lambda \mathrm{LC}$ and $\mathrm{IgD} / \mathrm{IgM}$ described in few reports, unfortunately no data concerning influence of coexistence of two and more patterns on renal outcome is available [22-30].

Here we present a case of IgD myeloma, manifested with the combination of LCDD and cast nephropathy.

\section{Caucasian Male, 52 Years Old, Admitted July 14, 2015.}

Main Complains: Weakness, dizziness, breath shortage, loin pain.

Previous Medical History: In 1988 he was diagnosed with hepatitis B, no treatment, no follow-up for many years. In 2010, his nose basalioma was removed in the outpatient clinic, preoperative work-up was unremarkable.

History of Present Illness: March 2015 he developed unexplained high grade fever, spontaneously resolved a week later, since that time felt weakness, palpitations, dyspnea, and diffuse abdominal pain. His BP was $120 / 80 \mathrm{~mm} \mathrm{Hg}$, HR 98bpm. Work-up in outpatient clinic (May 2015) showed Hb 13.1g/dL, Plt $222 \times 10^{9} / \mathrm{L}$, ESR $14 \mathrm{~mm} / \mathrm{h}$, creatinine $439 \mu \mathrm{mol} / \mathrm{L}(4.96 \mathrm{mg} /$ $\mathrm{dL}$ ], urea $20.8 \mathrm{mmol} / \mathrm{L}(125 \mathrm{mg} / \mathrm{dL}) \mathrm{K}+5.9 \mathrm{mmol} / \mathrm{L}$, AsAT $35 \mathrm{U} / \mathrm{L}$, AlAT 50U/L, GGTP 135U/L, AP 113U/L, proteinuria 1,03g/L with normal urinary sediment. Gastroscopy found 2 gastric and duodenal ulcers. He was taking omeprazole and beta-blockers without any improvement.

June 2015 he was admitted to the nephrology unit of local hospital with anemia ( $\mathrm{Hb} 9.4 \mathrm{~g} / \mathrm{dL}$ ), thrombocytopenia (Plt $106 \times 10^{9} / \mathrm{L}$ ) and progressive decline of kidney function with hyperkalemia and acidosis (creatinine $634 \mu \mathrm{mol} / \mathrm{L}$ [7.17md/dL], urea $26 \mathrm{mmol} / \mathrm{L}$ [156mg/dL], uric acid $520 \mu \mathrm{mol} / \mathrm{L}[8.7 \mathrm{mg} / \mathrm{dL}]$, phosphate $2.2 \mathrm{mmol} / \mathrm{L}, \mathrm{K}+6.5 \mathrm{mmol} / \mathrm{L}, \mathrm{pH} 7.27, \mathrm{BE}-12,9 \mathrm{mmol} / \mathrm{L}$ ) Proteinuria was still moderate $(1.08 \mathrm{~g} /$ day $)$, urinary sediment - unremarkable. WBC count, glucose, bilirubin, total protein, albumin, cholesterol, $\mathrm{LDH}$, serum $\mathrm{Fe}, \mathrm{Na}+\mathrm{Ca}++$ and lactate were normal. ECG and ECHO-CG showed both left and right ventricular hypertrophy; abdomen ultrasound revealed single portal lymph node $12 \times 9 \mathrm{~mm}$, other findings were unremarkable. Kidney ultrasound showed enlarged kidneys - both $128 \times 54 \mathrm{~mm}$, parenchyma $22 \mathrm{~mm}$, without any other significant changes; chest CT found slight enlargement of mediastinal lymph nodes (max $13 \mathrm{~mm})$.

Additional work-up found low IgA, IgG and IgM levels, cryoglobulinemia $1+$, and high CRP $(23,2 \mathrm{mg} / \mathrm{L})$. Serum protein electrophoresis showed M-spike 3.4\%-2.2g/L in $\gamma$-zone. IgE, RF, C-3, C-4, ANA, ANCA and PTH titers were within normal range. Anti-HBV-core IgG, anti-HCV IgG/IgM, anti-HCV-core IgM, and antiHCV-NS3antigen were positive, other HBV and HCV serum tests - negative. PCR HBV found viral load $<750$ copies/mL, PCR HCV - viral load $4 \times 10^{6}$ copies/mL, HCV genotype turned to be $3 \mathrm{a} / 3 \mathrm{~b}$.

He was suspected with rapidly-progressive GN, possibly cryoglobulinemic, and underwent kidney biopsy. According to the pathology report: Light Microscopy (LM) with standard staining's showed 8 hypertrophied glomeruli with marked hypercellularity (capillary WBC infiltration); interstitial fibrosis with prominent lymphohistiocytic infiltration and tubular atrophy about 30\%; massive hyaline casts in preserved tubular lumen; and oedematous arterial walls. Additional Congo red staining for amyloid was negative. Immune staining's were negative for IgA, IgG, IgM,C3 and $\kappa \mathrm{LC}$, and strongly positive for $\lambda$ LC - linear deposits along Glomerular Basement Membrane (GBM) and Tubular Basement Membrane (TBM) and also $\lambda$ LC deposition in casts. Electron microscopy (EM) confirmed dense fine granular GBM and TBM deposits.

That was interpreted as the LCDD, and bone marrow aspiration in search for LPD was performed. Bone marrow smear showed $1.8 \%$ of plasma cells and $22.9 \%$ of lymphocytes (probably due to peripheral blood admixture). Immunophenotyping of bone marrow cells found plasma cell phenotype CD45+dim, CD38+(high expression), CD138+, CD56-, CD28+, CD200-, CD117-, CD20+.

He was seen by a local hematologist, diagnosis of multiple myeloma was declined, and patient was diagnosed with "primary" LCDD. Serum and urine immunochemistry were ordered, and the patient was referred to our clinic for the second opinion.

At Admission: Conscious, alert, slightly depressed. Body temperature $37,2^{\circ} \mathrm{C}$, RR 18 per minute, pulse regular 98 per minute, BP 140/90mm Hg. Pale, well nourished, low extremities mild oedema. HEENT and neck otherwise normal. No palpable peripheral lymph nodes. Lungs: no dullness to percussion, any rhonchi, wheezes or rubs. Heart: regular rhythm, no murmur. Abdomen soft, non-tender, slightly tympanitic, bowel sounds normal. Liver $+2 \mathrm{~cm}$ below rib arch, non-painful, spleen and kidneys not felt. Urination is free, urine normally coloured, and urine output was about $1000 \mathrm{ml} /$ day.

Express Work-Up: Creatinine $1100 \mu \mathrm{mol} / \mathrm{L}$ [12.4mg/ $\mathrm{dL}$, $\mathrm{pH}$ 7.25, $\mathrm{K}+5.8 \mathrm{mmol} / \mathrm{L}$, urine Bence-Jones protein positive. Ultrasound: significantly enlarged kidneys (148x66 and $142 \times 68 \mathrm{~mm}$, parenchyma $25 \mathrm{~mm}$ ), hepatosplenomegaly, periportal lymphadenopathy, enlarged $(12,8$ and $6 \mathrm{~mm})$ right axillary lymph nodes with hypoechogenicity and compromised differentiation. 
Diagnostics Considerations, Treatment and Further Work-Up: At that point we diagnosed AKI, and concluded that the cause of AKI could be nothing but cast nephropathy on top of LCDD in a patient with myeloma. Patient was urgently started with hemodialysis and normal saline infusions, skeletal X-ray, peripheral lymph nodes ultrasound, and bone marrow biopsy were performed, and immunochemistry results ordered from external lab, and kidney biopsy paraffin blocks were reprocessed and re-evaluated.

Skeletal X-ray: Did not found any lesions.

Immunochemistry: Showed traces of paraprotein D- $\lambda$ and Bence-Jones- $\lambda$ in serum, and urinary excretion of BenceJones- $\lambda 1.22 \mathrm{~g} /$ day. Cryoglobulins were not found IgG, IgA and $\kappa / \lambda$ coefficients were below the normal range, CRP and $\beta 2$ microglobulin- significantly elevated.

Kidney biopsy re-processing and re-assessment (Figures 1-6): Sections of formalin fixed paraffin-embedded tissue were stained with Masson's trichrome, periodic acid-Schiff, and Jones' silver for LM. 12 glomeruli, slightly enlarged, with normal capillary wall, mild mesangial widening without mesangial or endocapilary proliferation. Total acute tubular necrosis with multiple large tubular casts, PAS- and Jones-negative, and fuxinpositive on Masson's staining, surrounded by giant polynuclear cells. Severe tubulo-interstitial infiltration with lymphocytes,

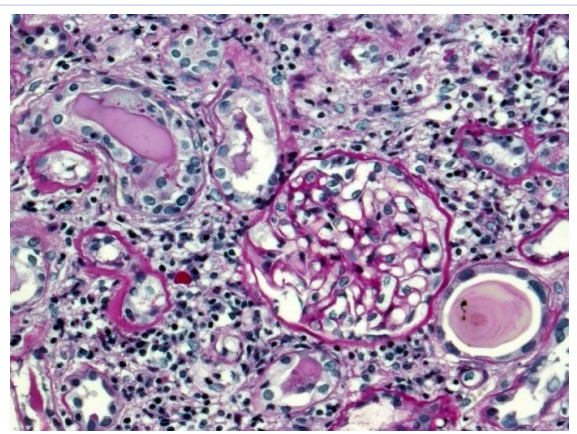

Figure 1: Light microscopy. PAS-negative and slightly positive casts with peripheral cell reaction, acute tubular injury and moderate tubulointerstitial inflammation. Normal glomerulus. Periodic acid-Schiff $\mathrm{x} 200$.

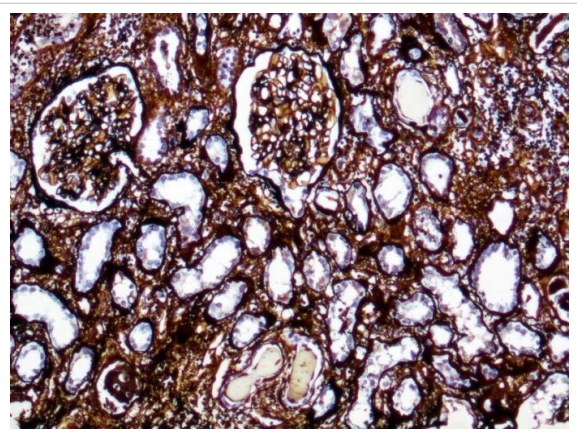

Figure 2: Light microscopy. Cortex fragment with agiro-negative casts in tubular lumen, acute tubular injury and slight glomerulomegaly. Jones silver impregnation $\times 100$.

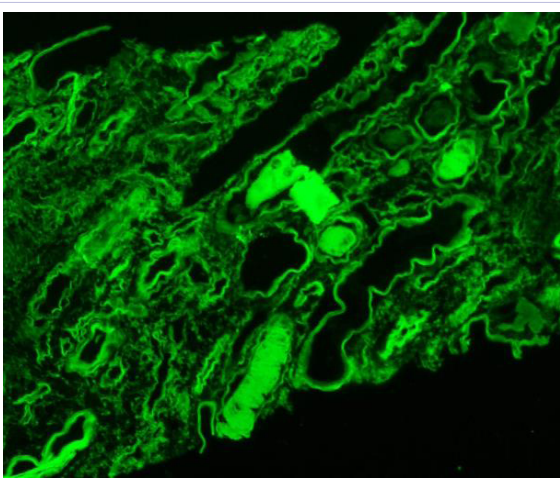

Figure 3: Immunofluorescence. Medulla fragment with $\lambda$-positive casts and diffuse linear expression of $\lambda \mathrm{LC}$ along tubular basement membrane and smooth-muscle walls of small arteries and arterioles; $\mathrm{x} 100$.

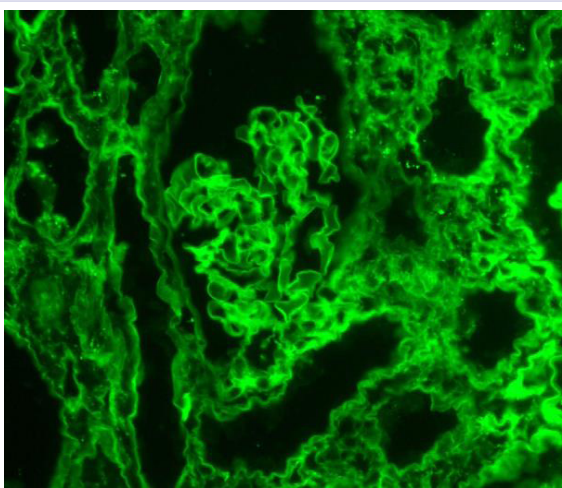

Figure 4: Immunofluorescence. Diffuse linear expression of $\lambda \mathrm{LC}$ along glomerular basement membrane and tubular basement membranes; $\mathrm{x} 200$.

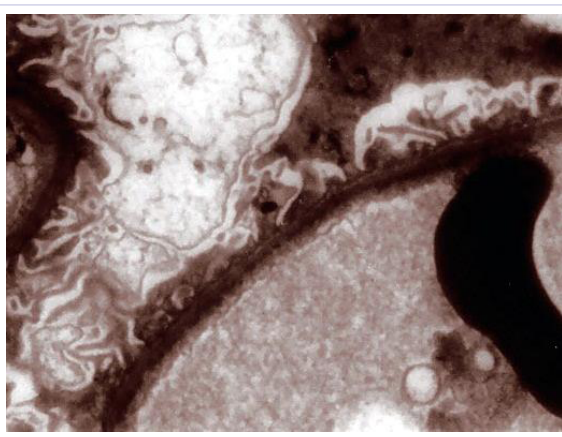

Figure 5: Electron microphotograph. Diffuse fine granular intramembranous dense deposits in glomerular basement membrane.

plasma cells and neutrophils, most prominent in zones of casts accumulation. Diffuse tubular atrophy and interstitial fibrosis about $40 \%$. Arterioles and small arterial walls thickened due to muscular layer hypertrophy. Immunofluorescence on formalin fixed/paraffin embedded sections with FITC-conjugated anti IgA, IgG, IgM, C1q, C3, fibrinogen, $\lambda$ and $\kappa$ LC antibodies showed diffuse linear expression $4+$ for $\lambda$ LC along all basal membranes - glomerular, tubular, arteriolar and arterial, and also 4+ $\lambda$ LC expression in the casts. All other immune stainings were negative. EM: toluidine-blue semi-thin sections showed 1 otherwise normal 


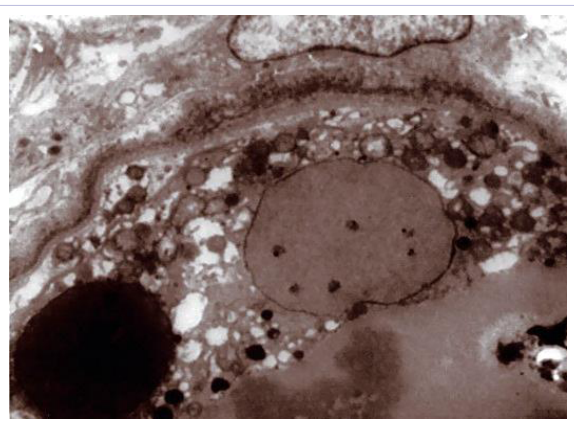

Figure 6: Electron microphotograph. Diffuse fine granular intramembranous dense deposits in tubular basement membrane.

glomerulus, and tubule containing large cast with peripheral cell reaction. Electron microphotographs demonstrated diffuse fine granular intramembranous dense deposits in GBM and TBM. Conclusion: combined paraproteinemic nephropathy - castnephropathy plus LCDD.

Bone marrow biopsy: Revealed enhanced number of plasma cells by LM. Immunohistochemistry found most plasma cells expressing CD138,$+ \lambda$ LC restriction, focal expression of CD56+, and few plasma cells expressing CD19 (aberrant immunophenotype of plasma cell population).

Final Diagnosis: Multiple myeloma $\operatorname{IgD} \lambda$ and $\mathrm{BJ} \lambda$, cast nephropathy combined with LCDD; AKI, treated with HD; anaemia, thrombocytopenia, hepatosplenomegaly, peripheral, mediastinal and periportal lymphadenopathy, secondary immunodeficiency.

TreatmentandFollow-Up: Patientwasseen by haematologist and referred to haematology unit for chemotherapy. At the latest evaluation (September15, 2015) patient receiving standard chemotherapy per BCD (Bortezomib-CyclophosphamideDexamethasone) protocol, doing well, but still on hemodialysis.

\section{Conclusions}

Presented case demonstrates characteristic features if IgD myeloma, like relatively young age, low grade IgD paraproteinemia, overproduction of LC with LC-proteinuria, lymphadenopathy and renal failure at presentation. Pathology findings with typical picture of LCDD were misleading, and only rapidly-progressive renal failure with kidney enlargement, not consistent with LCDD natural course, demanded re-assessment of kidney biopsy and more detailed work-up, which finally gave a clue to the diagnosis. The pathology pattern of renal damage: a combination of cast nephropathy and LCDD confirms the leading role of cast-nephropathy in the clinical presentation with AKI, dominating over other symptoms. According to our experience such combined renal damage is rare, but not unique -in our cohort of 139 patients with LPD and renal damage, confirmed by pathology, we also have patients with AL amyloidosis and LCDD, and with combination of cast nephropathy, AL amyloidosis and LCDD. This case also illustrates the importance of renal damage pattern, typical for MM, for diagnostics of this disease in patients without clinical MM features.

\section{References}

1. Sanders PW, Booker BB. Pathobiology of cast nephropathy from human Bence Jones proteins. J Clin Invest. 1992;89(2):630-9.

2. Cohen AH. The kidney in plasma cell dyscrasias: Bence Jones cast nephropathy and light chain deposition disease. Am J Kidney Di. 1998;32(3):529-32

3. Ronco PM. Paraneoplastic glomerulopathies: new insights into an old entity. Kidney Int. 1999;56(1):355-77.

4. Da'as N, Polliack A, Cohen Y, Amir G, Darmon D, Kleinman Y, et al. Kidney involvement and renal manifestations in non-Hodgkin's lymphoma and lymphocytic leukemia: a retrospective study in 700 patients. Eur J Haematol. 2001;67(3):158-6.

5. Hagler KT, Lynch JW Jr. Paraneoplastic manifestations of lymphoma. Clin Lymphoma. 2004;5(1);29-36

6. Herrera GA, Joseph L, Gu X, Hough A, Barlogie B. Renal pathological spectrum in an autopsy series of patients with plasma cell dyscrasia. Arch Pathol Lab Med. 2004;128(8):875-9.

7. Keeling J, Teng J, Herrera GA. AL-amyloidosis and light-chain deposition disease light chains induce divergent phenotypic transformations of human mesangial cells. Lab Invest, 2004(10);84(10):1322-38.

8. Nasr SH, Markowitz GS, Stokes MB, Seshan SV, Valderrama E, Appel GB, et al. Proliferative glomerulonephritis with monoclonal IgG deposits; a distinct entity mimicking immune-complex glomerulonephritis. Kidney Int, 2004;65(1):85-96.

9. Korbet SM, Schwartz MM. Multiple myeloma. J Am SocNephrol, 2006;17(9);2533-45.

10. Merlini G, Pozzi C. Mechanisms of renal damage in plasma cell dyscrasias: an overview. Herrera GA (ed):The Kidney in Plasma Cell Dyscrasias. ContribNephrol. Basel, Karger,vol 2007;153:66-86.

11. Ronco P, Plaisier E, Aucouturier P. Ig-related renal disease in lymphoplasmacytic disorders: An update. SeminNephrol. 2009;30:557-69. doi: 10.1016/j.semnephrol.2010.09.004.

12. Heher EC, Goes NB, Spitzer TR, Raje NS, Humphreys BD, Anderson KC, et al. Kidney disease associated with plasma cell dyscrasias. Blood, 2010;116:1397-404. doi: 10.1182/blood-2010-03-258608.

13. Cambier JF, RoncoP. Onco-nephrology: glomerular diseases with cancer. Clin J Am SocNephrol. 2012;7(10): 1701-12. doi:10.2215/ CJN.03770412.

14. Ben-Bassat I, Frand UI, Isersky C, Ramot B. Plasma cell leukemia with IgDparaprotein. Arch Intern Med. 1968;121(4):361-4.

15. Jancelewicz Z, Takatsuki K, Sugai S, Pruzanski W. IgD multiple myeloma. Review of 133 cases. Arch Intern Med. 1975;135:87-93.

16. Homan H, Ohtubo Y, Ito H, Azuma K, Yasumoto Y, Ohtuka H, et al. IgD multiple myeloma with renal involvement: case report. Jpn J Med. 1990;29:212-5.

17. Shimamoto Y, Anami Y, Yamaguchi M. A new risk grouping for IgD myeloma based on analysis of 165 Japanese patients. Eur J Hematol. 1991;47:262-7.

18. Blade J, Lust JA and Kyle RA. Immunoglobulin D multiple myeloma: presenting features, response to therapy, and survival in series of 53 cases. J ClinOncol. 1994;12:2398-1404.

19. BladeJ, Kyle RA. Nonsecretory myeloma, immunoglobulin D myeloma and plasma cell leukemia. Hematol Oncol Clin North Am. 1999;13:1259-71. 
20. Sinclair D. IgD myeloma: clinical, biological and laboratory features. Clin Lab. 2002;48(11012):617-22.

21. Zagouri F, Kastritis E, Symeonidis AS, Giannakoulas N, Katodritou E, Delimpasi S, et al. Immunoglobulin D myeloma: clinical features and outcome in the era of novel agents. Eur J Hematol. 2015;92(4):308312. Doi:10.1111/ejh.12255.

22. Kaplan B, Vidal R, Kumar A, Ghiso J, Frangione B, GalloG. Aminoterminal identity of co-existent amyloid and non-amyloid immunoglobulin kappa light chain deposits. A human disease to study alterations of protein conformation. ClinExpImmunol. 1997;110:47278.

23. Buxbaum J, Gallo G. Nonamyloidotic monoclonal immunoglobulin deposition disease. Light-chain, heavy-chain, and light-and heavychain deposition diseases. HematolOncolClin North Am. 1999;13:1235-48.

24. Kyle RA, Gertz MA, Witzig TE, Lust JA, Lacy MQ, Dispenzieri A, et al. Review of 1027 patients with newly diagnosed multiple myeloma. Mayo Clin Proc. 2003;78(1):21-33.

25. Tharp AM, Woodruff RD ,Shihabi ZK. IgD-kappa myeloma: an unusual case. Annals of Clin and Lab Science. 2003;33(1):97-100

26. Gokden N, Cetin N, Colakoglu N, Kumar J, Abul-Ezz S, Barlogie B, Liapis
$\mathrm{H}$, Walker PD, et al. Morphologic manifestations of combined lightchain deposition disease and light-chain cast nephropathy. Ultrastruct Pathol. 2007;31(2):141-9.

27. Qian Q, Leung N, Theis JD, Dogan A, Sethi S. Coexistence of myeloma cast nephropathy, light chain deposition disease, and nonamyloid fibrils in a patient with multiple myeloma. Am J Kidney Dis. 2010;56(5):971976. doi: 10.1053/j.ajkd.2010.06.018.

28. Lorenz EC, Sethi S, Poshusta TL, Ramirez-Alvarado M, Kumar S, Lager DJ, et al. Renal failure due to combined cast nephropathy, amyloidosis and light-chain deposition disease. 2010; Nephrol Dial Transplant. 25:1340-3 doi:10.1093/ndt/gfp735.

29. Hada R, Poudyal B, Sharma A, Khatri R. Lambda Light Chain Myeloma with Oliguric Cast Nephropathy and Remission with Bortezomib, Doxorubicin and Dexamethasone. J Nepal Med Asoc. 2012;52(188):192-5.

30.Chen ZW, Kotsikogianni I, Raval JS, Roth CG, Rollins-Raval MA. Biclonal IgD and IgM plasma cell myeloma: a report of two cases and literature review. Case Reports in Hematology. 2013;2013:293150 doi.org/10.1155/2013/293150. 